\title{
دمج كلمات عربية لتشكيل مصطلحات علمية
}

\section{Combining Arabic words to create scientific terms}

Khaled Moustafa ${ }^{1}$ خالا مصطفى

ملخص

تتسم اللغة العربية بضعف المحتوى العلمي والاصطلاحي، مقارنة مع اللغات ذات الجذور الهندو أوربية واللاتينية، لعدم وجود طريقة سهلة ومعتمدة للاشتقاق العلمي بما يتلاءم مع خصائص وطبيعة اللسان العربي. في هذه المقالة، سيتم اقتراح طريقة جديدة للاشتقاق العلمي بناءً على دمج عدة كلمات عربية في كلمة واحدة، ثم استخدام علامة تشكيل عمودية جديدة أو فاصلة عليا (') تستخدم لهذا الغرض كعلامة دمج عندما تكون في وسط الكلمة، وكعلامة اثتقاق عندما تكون في آخرها. مثال: يمكن كتابة عبارة "حمض وراثي" ضمن كلمة واحدة ك (حمض'وراثي) بوجود الفاصلة العليا وسط الكلمة الجديدة، بدلاً من كلمتين منفصلتين (حمض وراثي)، وبمكن اختصار عبارة مضاد حيوي ك (مح')، حيث تشير الفاصلة العليا في نهاية الكلمة على أنها اشتقاق من أوائل حروف كلمتي "مضاد حيوي". وهكذا، يمكن إثراء القاموس العلمي العربي بمصطلحات واشتقاقات علمية جديدة ناتجة عن دمج عدة كلمات في كلمة واحدة في شتى ميادين المعرفة لحل معضلة ندرة المصطلحات العلمية والطبية في اللغة العربية، وصعوبة تركيبها واشتقاقها.

\section{Abstract}

Scientific terms/acronyms in Arabic are currently rare and difficult to construct compared with English due to the absence of easy approach to create them. A new method will be proposed here to facilitate the introduction of new scientific terms in Arabic by the combination of two or more Arabic words in one word as a new scientific term by using a new vertical dash (or apostrophe) as a combination mark. A scientific expression composed of several words can thus be merged and written in one Arabic word only with a vertical dash between the last letter of the first word and the first letter of the next word and so on. This might help circumvent the paucity of scientific terms in Arabic in the various fields.

\section{Résumé}

\footnotetext{
محرر الأرشيف العربي العلمي Editor of Arabic Science Archive (arabixiv.org). Email: khaled.moustafa@arabixiv.org
} 
Les termes scientifiques spécialisés en arabe sont relativement rares et difficiles à construire par rapport à l'anglais en raison de l'absence d'une approche simple et efficace. Une nouvelle méthode sera proposée ici pour faciliter la création de nouveaux termes scientifiques en combinant deux ou plusieurs mots arabes en un seul en utilisant un nouveau tiret vertical (ou apostrophe) à placer à l'intérieur du nouveau mot, comme une marque de combinaison lexicale. Une expression scientifique longue de plusieurs mots peut donc être fusionnée en un seul mot long, écrit en tant que tel, à l'aide d'un tiret vertical entre la dernière lettre du premier mot combiné et la première lettre du second mot, et ainsi de suite. Cela peut contribuer à l'enrichissement de la langue arabe de nouveaux termes scientifiques d'une manière simple et facile à mettre en œuvre.

للاختصار العلمي جوانب إيجابية كثيرة في بعض الحالات لاختزال أو نحت كلمات طويلة وتقصير لفظها وتسهيل حفظها وتوفير مساحة طبعها. ويتذمر الكثير من الدارسين العرب مما يزعمون عن عدم ملاعمة اللغة العربية للاختصار والاشتقاق العلمي مقارنة مع اللغات الهندو أوربية واللاتينية، التي تتميز بسهولة الاختصار والاشتقاق بسبب طريقة كتابتها بحروف منفصلة، وهو ما تفتقر إليه اللغة العربية. ولكن بغض النظر عن تحيز هذا الادعاء ضد اللغة العربية وعدم صحته بتاناً، يمكن الاعتماد على خاصية الوصل في اللغة العربية لجعلها ميزة لا عيباً، لوصل أو دمج عدة كلمات ضمن كلمة واحدة لتكوين مصطلحات جديدة مركبة تتلاعم مع طبيعة اللغة العربية المتصلة حروفها.

في مقالة سابقة [1]، تم اقتراح إدخال علامة تشكيل جديدة عمودية الشكل توضع على الحرف الأخير من كلمات مختصرة، لتعريفها على أنها مصطلحات علمية مركبة من دمج الحروف الأولى لتعابير طويلة. ولنفس الغاية، سيتم هنا اقتراح طريقة مكملة باستخدام نفس الثرطة العمودية، كعلامة دمج بين عدة الكلمات في كلمة واحدة أو تعبير واحد لتعزيز طرق الاثتقاق العلمي. على سبيل المثال، يمكن كتابة عبارة "هاتف محمول" في كلمة واحدة ك "هاتفحمول" و "حمض وراثي" أو "حمض نووي" ك "حمضوراثي" أو "حمضنووي" نباعاً، بوجود علامة الدمج في منتصف الكلمة، بدلاً من كتابتهما منفصلتين، كما هو موضح في الشكل 1. بمعنى آخر، إذا كان الدمج ناتجاً عن كلمتين، توضع الثنََُّْة العمودية بين الحرف الأخير من الكلمة الأولى وبين الحرف الأول من الكلمة الثانية، وإذا كان الدمج بين ثلاث كلمات أو أكثر، توضع الثَُّطَة العمودية دائماً بين الحروف الدامجة، أي، بين الحرف الأخير من الكلمة الأولى والحرف الأول من الكلمة التي تليها، وهكذا دواليك. 
لا ريب أن عملية الدمج بين كلمات مختلفة، كما في الثكل 1، ستُتج تعابيراً لغوية طويلة أو غريبة نوعاً ما ظاهرياً، ولكن بالتكرار والاستخدام المتداول تصبح مألوفة وتزول غرابتها.

\begin{tabular}{|c|c|c|c|}
\hline صيغة الجمع & المصطلح العربي بعد الدمج علامة عمودية & المصطلح العربي & $\begin{array}{l}\text { English } \\
\text { term }\end{array}$ \\
\hline حموضُور اثية & حمضنور اثي & حمض وراثي / أو & $\left\{\begin{array}{c}\text { ribonucleic } \\
\text { acid }\end{array}\right.$ \\
\hline- & 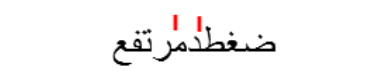 & ضغط دم مرتفع & hypertension \\
\hline مخزوناتؤراثية أو مخازنوراثية & مخزونور اثي & مخزون وراثي (جينوم) & genome \\
\hline بروتيناتكانسة & 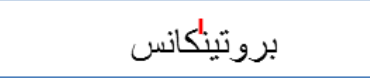 & بروتين كانس & scavenger protein \\
\hline- & بينشبكات & 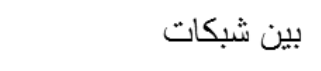 & internet \\
\hline
\end{tabular}

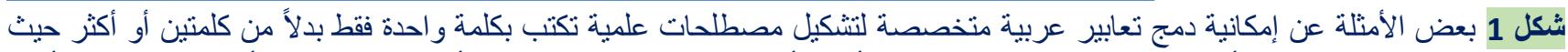

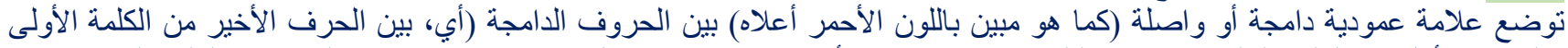

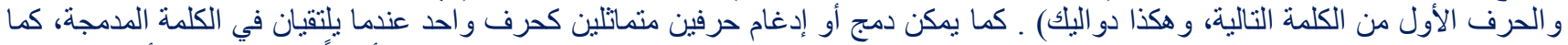

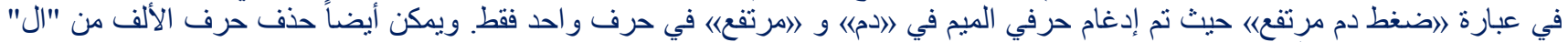

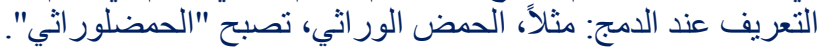

ولتطبيق هذه الطريقة عملياً، هناك حالياً صعوبة تقنية تتعلق بعدم إمكانية استخدام لوحات المفاتيح الحالية لإضافة علامة عمودية على أحد الحروف الداخلية لكلمة عربية دون أن يؤدي ذلك إلى فصل حروفها وتقسيمها. ولكن، إلى الكاله يمكن تلافي هذا العيب تقنياً من قبل مصنعي الحواسب الآلية لإدخال شَرْطة عمودية خاصة باللغة العربية، كعلامة دمج أو وصل بين الحروف العربية، ليتم استخدامها لذلك الغرض دون أن تؤدي إلى فصل الحروف، كما هو الحال

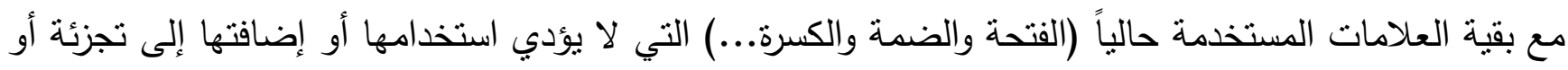
فصل الحروف بعضها عن بعض.

وهناك خيار آخر، ربما أسهل وأسرع تلاؤماً مع لوحات المفاتيح الحالية وهو دمج الكلمات واستخدام فاصلة عليا ضمن الكلمات المُدْمجة، كعلامة دمج للمصطلحات العلمية، دون أن يتم لصق الحروف كما هو مبين في الثكل 1 الثمل وإنما تقريبهم ووضع الفاصلة العليا كحد فاصل بين حروف الكلمات المدمجة، كما لو كانت كلمة واحدة مكونة من حروف منفصلة. على سبيل المثال، يمكن كتابة الكلمات التي تظهر في الثكل 1 كما يلي: 
هاتف'محمول أو حَمُولٌ بدلاً من "هاتف محمول" حيث يتم دمج "هاتق" و "محمول" في كلمة واحدة تفصلهما فاصلة عليا (تستخدم

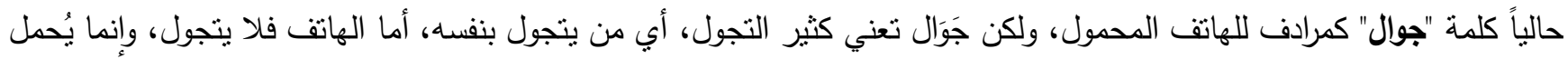
ويُّجول به، ولكنه لا يتجول بذاته لكي يقال عنه جوَال).

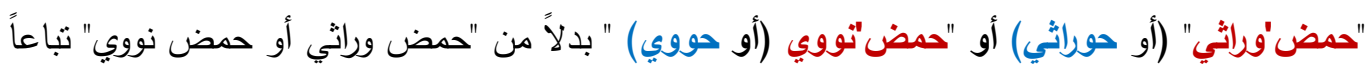

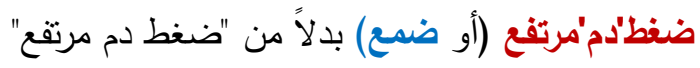

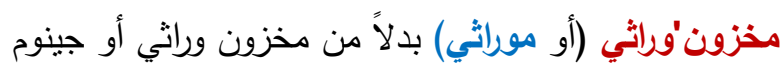
بروتين 'كانس (أو بروكانس) بداًا من بروتين كانس

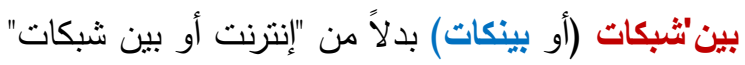

كما يمكن اختصار أو نحت مصطلحات علمية دون أي فاصل بين حروفها، كما هي الكلمات بالأزرق أعلاه

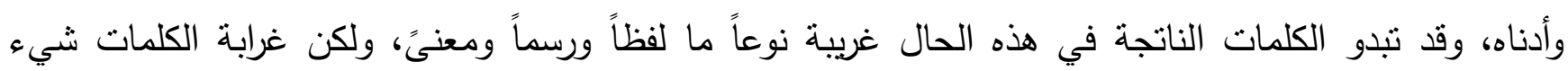
مألوف في اللغة العربية حتى في القرآن الكريم، مثل "كهيعص" و "عسق"، التي لا يُعرف معناها أو دلالتها بالضبط. كما أن اللغة العربية تحتوي للتو على كلمات كثيرة منحوتة من اختصار ودمج كلمات أخرى، مثل:

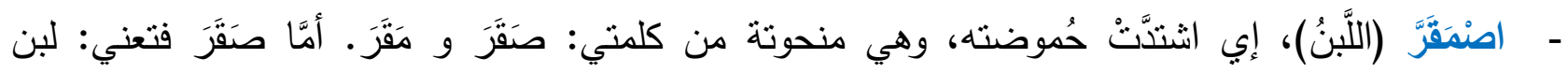

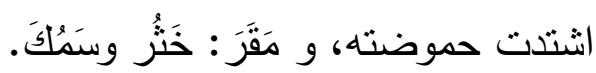

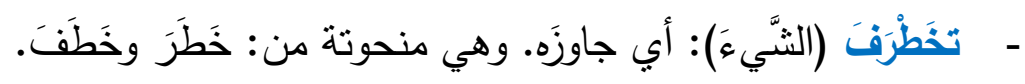

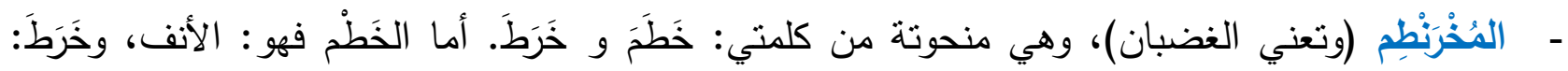

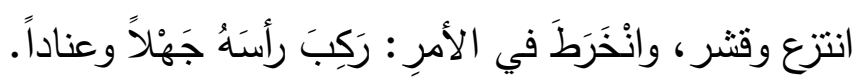
- ـ صَنْصَلِقِق (وتعني المرأة الصخّابة، أبي كثيرة الصخب وذات الصوت المرتفع) وهي منحوتة من: "صهل وصلق". - - خَزْعال (ناقة خزعال، أبي بها عَرَجُ)، وهي مشتقة من: خَزَل، أي تثاقل في مشيه وكأن في قدمه شوكُّ، وخَزعَ، أبي قطع أو نَخَلّفَ في المسبر عن الركَبْ.

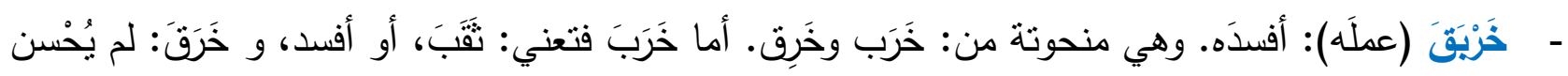
في العمل، ومنه الأخرقَ: الذي لا يُحسِن عمله.

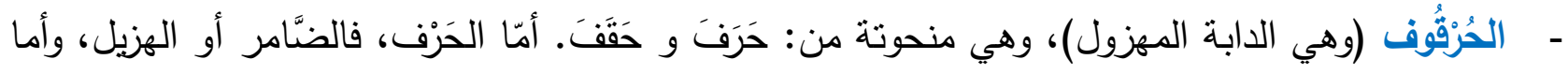

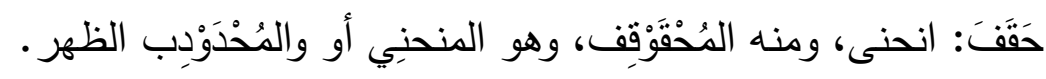




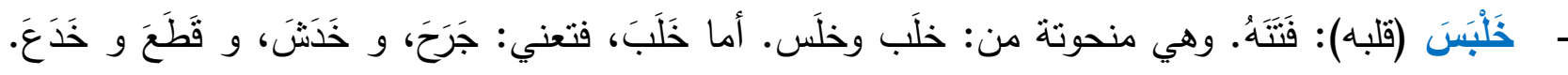
أما خَلَسَ فتعني: أخذ أو سلب في مخاتلة ونهزة أو انتهاز .

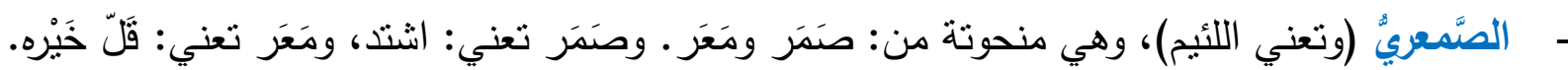

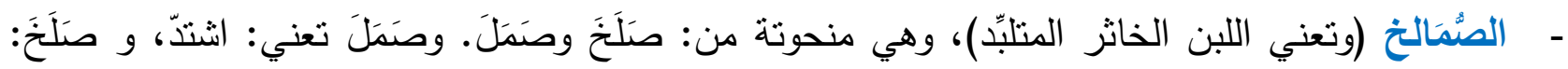

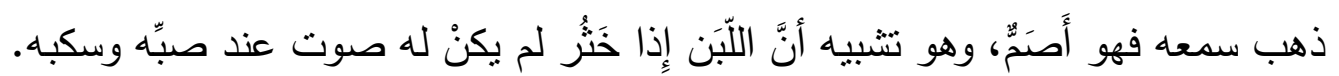

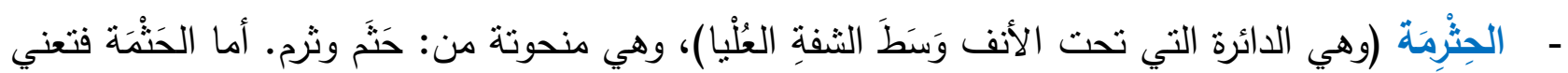

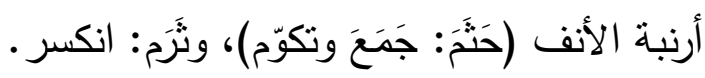

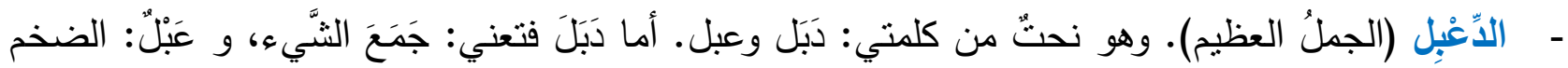
من كل شيء.

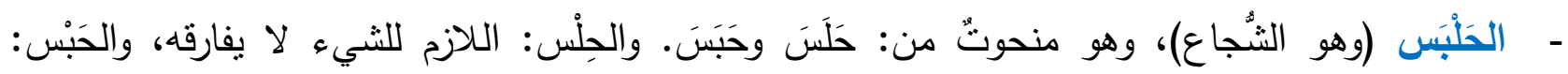
الثنجاعة، والحجز والإمساك أيضاً.

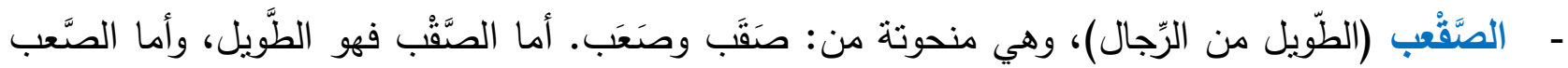
فمن الصُّعوبة.

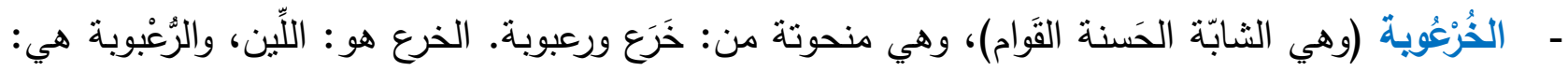
الناعمة.

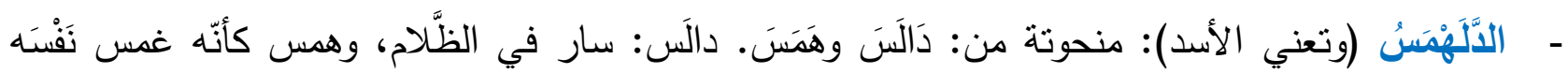

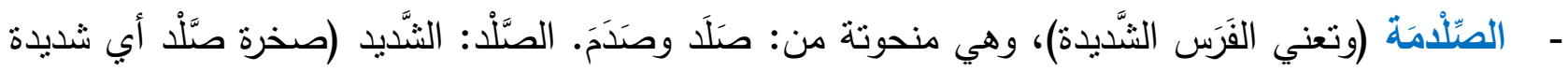
وقاسية)، وصَدَمَ: ارتطم بالثيءء وفي هذا يقول امرؤ القيس واصفاً فرسه:

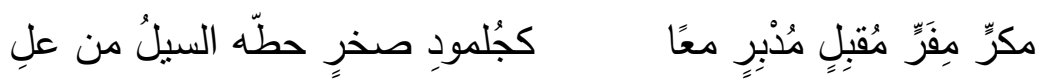

تدل هذه الكلمات وغيرها كثير على إمكانية النحت والاشتقاق العلمي في اللغة العربية أسوة بغيرها من اللغات. وفي بعض اللغات الأوربية، كالألمانية والسويدية، يمكن تتكيل كلمات طويلة للغاية، مكونة من دمج ثلاث أو أربع أو خمس كلمات معاً في كلمة واحدة قد يصل طولها إلى بضع عشرات من الحروف. أما في العربية، فإن أطول الكلمات المعروفة حالياً قد لا يتجاوز عدد حروفها عشرة إلى عشرين حرفاً فقط في أفضل الحالات. لذلك، فإن طريقة الدمج المقترحة هنا سنساعد على تشكيل مصطلحات عربية متخصصة بالمجالات العلمية والتقنية للمساهمة 
في تكريس الاشتقاق العلمي الذي تفتقر إليه اللغة العربية بشكل كبير • وبذلك، قد يُصبح الاختصار العلمي في الميادين العلمية العربية أمراً مبسطاً نوعاً ما، وخاضعاً لضوابط واضحة ودقيقة، حيث تكون الفاصلة العليا أو علامة التشكيل العمودية المقترحة سابقاً [1] دليلاً على أن الكلمة التي تحتوبها هي مصطلح علمي متخصص ناتج عن دمج الكلمات التي تكونها، لكيلا يُستغرب معناها ولفظها وكتابتها. وإذا التبس الأمر، وجُهِل معناها بعد الدمج، فيمكن ردها إلى أصلها قبل الدمج من خلال تجزئة اللفظ مقطعياً قبل وبعد العلامة العمودية (أو الفاصلة العليا) التي ستشكل علامة لفظ مقطعي بين ما قبلها وما بعدها في الكلمة الجديدة (الناتجة عن الدمج) فيسهل الرجوع إلى الأصل عند الالتباس.

بعبارة أخرى، يمكن استخدام فاصلة عليا وسط كلمة عربية كعلامة دمج وفي آخرها كعلامة اشتقاق. مثلاً، نثير الفاصلة العليا في (حمض'وراثي أو هاتف'محمول أو بروتين'كانس) على أن هذه الكلمات مصطلحات ناتجة عن آن

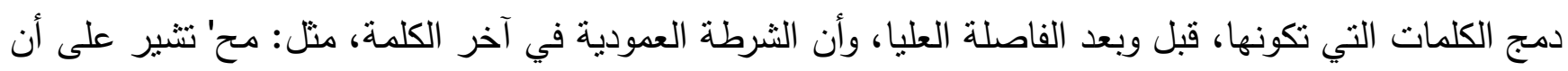
هذه الكلمة هي اشتقاق من أوائل حروف كلمتي "مضاد حيوي".

وهكذا، باستخدام شرطة عمودية (أو فاصلة عليا) في آخر الكلمة كعلامة نحت [1]، وشرطة عمودية أو فاصلة عليا وسط الكلمة كعلامة دمج، كما هو مقترح هنا، يمكن بهاتين الطريقتين المتكاملتين تكوين واعتماد مصطلحات علمية عربية في شتى المبادين. كما يمكن اعتماد الدمج أو الوصل أو النحت بين الكلمات العربية دون علامة دمج عمودية أو اشتقاق، كما في الأمتلة أعلاه (باللون الأزرق)، ولكن في هذه الحال قد يكون من الصعب قراءة الكلمة

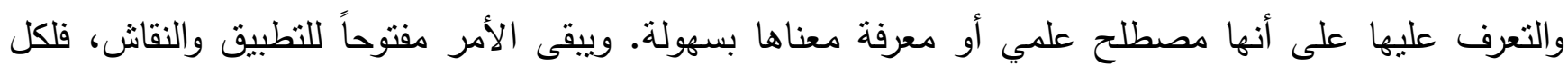
طريقة ميزات وعيوب، وليس هناك طريقة مثالية وعامة تكون كلها مزايا دون عيوب.

كلمات مفتاحية: اللغة العربية والاثتقاق العلمي، مصطلحات علمية، اثتقاق علمي، علامات التشكيل، حركات التشكيل، دمج الكلمات العربية، دمج المصطلحات العلمية، النحت اللغوي

\section{References}

مراجع

1. Moustafa, K., علامة تشكيل جديدة لتسهيل الاختصار العلمي في اللغة العربية. Arabic Science Archive, 2019. DOI: https://doi.org/10.31221/osf.io/dauqk. 